\title{
A new insight into the oxidative mechanism of caffeine and related methylxanthines in aprotic medium: May caffeine be really considered as an antioxidant?
}

\author{
Rita Petrucci $^{\mathrm{a}, *}$, Giuseppe Zollo ${ }^{\mathrm{a}}$, Antonella Curullib ${ }^{\mathrm{b}}$, Giancarlo Marrosu ${ }^{\mathrm{a}}$ \\ a Dipartimento di Scienze di Base e Applicate per l'Ingegneria SBAI, Sapienza Università di Roma, via del Castro Laurenziano 7, I-00161 Rome, Italy \\ b CNR ISMN Istituto per lo Studio dei Materiali Nanostrutturati UOS Sapienza, Via del Castro Laurenziano 7, I-00161 Roma, Italy
}

\section{A R T I C L E IN F O}

\section{Keywords:}

Caffeine

Methylxanthines

Cyclic voltammetry

UV-vis spectroelectrochemistry

DFT

Reactive Oxygen Species

\begin{abstract}
A B S T R A C T
Background: Antioxidant properties have been recently suggested for caffeine that seems showing protective effects against damages caused by oxidative stress. In particular, a HO^ scavenging activity has been ascribed to caffeine. Even if the oxidation of caffeine has been widely studied, the antioxidant mechanism is still far to be understood.

Methods: The electrochemical behavior of caffeine, theobromine and theophylline was studied in aprotic medium by cyclic voltammetry and electrolysis in UV-vis cell; a computational analysis of the molecular structures based on the Density Functional Theory was performed; the reactivity of all substrates towards lead dioxide, superoxide and galvinoxyl radical was followed by UV-vis spectrophotometry.

Results: Results supported the mono-electronic oxidation of the $\mathrm{C}^{4}=\mathrm{C}^{5}$ bond for all substrates at high oxidation potentials, the electron-transfer process leading to a radical cation or a neutral radical according to the starting methylxanthine $\mathrm{N}^{7}$-substituted (caffeine and theobromine) or $\mathrm{N}^{7}$-unsubstituted (theophylline), respectively. A different following chemical fate might be predicted for the radical cation or the neutral radical. No interaction was evidenced towards the tested reactive oxygen species.

Conclusions: No reactivity via H-atom transfer was evidenced for all studied compounds, suggesting that an antiradical activity should be excluded. Some reactivity only with strong oxidants could be predicted via electron-transfer. The acclaimed HO ${ }^{*}$ scavenging activity should be interpreted in these terms. The study suggested that CAF might be hardly considered an antioxidant.

General significance: Beyond the experimental methods used, the discussion of the present results might provide food for thought to the wide audience working on antioxidants.
\end{abstract}

\section{Introduction}

Among methylxanthines, caffeine (1,3,7-trimethylxanthine, CAF), theobromine (3,7-dimethylxanthine, $\mathrm{TBr}$ ) and theophylline (1,3-dimethylxantine, TPh) are widely distributed in nature, where are present in almost one hundred species of the Plant Kingdom, including coffee, cacao (the major natural source of $\mathrm{TBr}$ ) and tea (the major natural source of TPh) [1].

Thus methylxanthines have been included in human diet for a long time and are nowadays generally assumed in the diet because widespread in many products highly consumed all over the world, from traditional coffee, tea and chocolate, to soft and energy drinks and more recently as food supplements.

\footnotetext{
* Corresponding author.

Email address: rita.petrucci@uniroma1.it (R. Petrucci)
}

Many systemic effects in humans have been ascribed to methylxanthines, but mechanisms underlying their biological effects are not yet fully understood. Anyway, these relevant biological effects combined with a relatively low toxicity make these natural compounds pharmacological tools with increasing therapeutic potentialities [1].

CAF is by far the most studied methylxanthine. Both positive and negative physiological effects of CAF are well known, from stimulation of the central nervous system, diuresis and gastric acid secretion [2] to nausea, trembling and nervousness [3]. It is known to increase psychomotor performance [4], and because of its analgesic effects in headache and pain it is added in analgesic and antipyretic drug formulations [5,6]. Pharmacological effects of CAF and related methylxanthines on respiratory, cardiovascular, neurological and neurodegen- 
erative diseases as well as a positive impact on obesity, diabetes, fertility and cancer have been also widely reported in literature [1]. The beneficial effects of CAF and methylxanthines have been ascribed, at least in part, to a putative high antioxidant capacity. In fact, CAF has been also investigated for its potential antioxidant capacity towards oxidative damage and literature reports in vitro and in vivo studies evidencing some protective effects [7-9], mainly ascribed to a scavenging activity towards $\mathrm{HO}^{*}$ radicals $[10,11]$. Computational analysis based on the Density Functional Theory supported a strong HO• scavenging activity, while predicted modest, poor or no activity towards alkoxyl radical, $\mathrm{HOO}^{*}$ and $\mathrm{O}_{2}{ }^{--}$, respectively [12]. On the other hand, CAF resulted inactive towards moderate hydrogen/electron scavengers as DPPH and ABTS * $^{*}$ [11]. The term "antioxidant" is generally used for both antioxidant and antiradical activities, but while antioxidant activity represents the capability of a compound to inhibit an oxidative process, the antiradical activity represents the capability of a compound to react with free radicals, as clearly reported in literature [13]. As a consequence, antioxidant and antiradical activities occur through different molecular mechanisms, and compounds with antiradical properties might be ineffective as antioxidant and vice versa.

Further, experimental conditions of test systems for radical scavenging activity may strongly differ from in vivo environment. As an example, the hydroxyl radical scavenging activity measured by generating in situ high amounts of $\mathrm{HO}^{*}$, even if very popular as a method, has no practical meaning, actually, because of the very high reactivity of $\mathrm{HO}$ - with the most of substrates, including itself [13]. So, the understanding of the reactivity of a given compound, in terms of molecular mechanism, is required to explore and evaluate its antioxidant and/or antiradical potentialities.

Given the potentialities of CAF, any progress made in order to understand the molecular mechanisms underlying beneficial or harmful effects could meaningfully impact biomedical research.

Studies on the oxidation of CAF by radical species in water have been reported in literature and various mechanisms proposed [14-16]. Also the electrochemical oxidation of CAF in water has been studied since '70, and a pathway involving an irreversible overall four electrons process per molecule has been proposed $[17,18]$ : the oxidation was supposed starting from the imidazole $\mathrm{C}^{8}-\mathrm{N}^{9}$ double bond and yielding the methyl-uric acid first and the methyl-uric acid 4,5-diol as final product. The electrochemical oxidation of CAF in aprotic medium has been instead sparingly studied, only one report in literature, at least up to our knowledge [19]. Noteworthy, the aprotic medium can be considered more suitable to investigate mechanisms involving radical species, by reducing chemical reactions they might undergo. Further, due to both hydrophilicity and lipophilicity properties, CAF can distribute through all body fluids and cross all biological membranes when administered to humans or animals [1], and the aprotic medium provides an environment closer to the lipophilic one.

With the aim to get a deeper insight into the oxidative mechanisms CAF might undergo through under oxidative conditions, and to explore the potentialities of CAF as an antioxidant towards reactive oxygen species (ROS) main responsible of oxidative stress, the electrochemical behavior of CAF was studied in aprotic medium by cyclic voltammetry and controlled potential electrolysis, carried out in an UV-vis cell modified for spectroelectrochemistry. TBr and TPh were also studied under the same experimental conditions.

A computational analysis based on the Density Functional Theory (DFT) was performed on CAF and TPh molecular structure as a precious tool to interpret the electrochemical data.

The reactivity of $\mathrm{CAF}, \mathrm{TBr}$ and $\mathrm{TPh}$ towards reactive oxygen species as superoxide radical anion $\mathrm{O}_{2}{ }^{*-}$ and galvinoxyl free radical (G) was also investigated, and the reactions were followed by UV-vis spectrophotometry.

\section{Experimental}

\subsection{Materials}

Caffeine powder, ReagentPlus ${ }^{\circledR}$ (CAS Number 58-08-2), Theobromine analytical standard (CAS Number 83-67-0), Theophylline anhydrous, $\geq 99 \%$, powder (CAS Number 58-55-9), Galvinoxyl free radical (CAS Number 2370-18-5), 2,6-lutidine purified by re-distillation, $\geq 99 \%$ (CAS Number 108-48-5), dicyclohexano-18-crown-6, 98\% (CAS Number 16069-36-6), potassium superoxide, powder (CAS Number 12030-88-5), acetonitrile anhydrous, 99.8\%, <0.001\% water (CAS Number 75-05-8), $\mathrm{N}, \mathrm{N}$-Dimethylformamide anhydrous, $99.8 \%,<0.005 \%$ water (CAS Number 68-12-2) were purchased from Sigma-Aldrich and used without further purification.

Potassium superoxide was used as DMF saturated solution containing $0.15 \mathrm{molL}^{-1}$ dicyclohexano-18-crown- 6 , added to increase the solubility of $\mathrm{KO}_{2}\left(0.05 \mathrm{~mol} \mathrm{~L}^{-1}\right.$ final concentration) [20]. Tetraethylammonium perchlorate (TEAP) was purchased from $\mathrm{BDH}$ and dried under vacuum after crystallization. Water was deionized by Millipore Milli-Q Purification System. Lead dioxide $\mathrm{PbO}_{2}$ was freshly prepared [21].

\subsection{Methods \\ 2.2.1. Cyclic voltammetry}

Cyclic voltammetry was performed with a three-electrode multipolarograph AMEL 472 coupled with a digital $x / y$ recorder AMEL 863, using a working static glassy-carbon electrode (GC, $\varnothing=3 \mathrm{~mm}$ ), Ag-Ag$\mathrm{ClO}_{4}\left(0.1 \mathrm{~mol} \mathrm{~L}^{-1}\right) / \mathrm{AcN}$-fine porosity fritted glass disk-AcN $/ \mathrm{NaClO}_{4}$ $\left(0.1 \mathrm{molL}^{-1}\right)$-sintered glass disk [22] as reference and a platinum wire as counter electrode, in the range of scan rate $0.020-0.200 \mathrm{~V} \mathrm{~s}^{-1}$. The accuracy of the potential was $\pm 5 \mathrm{mV}$. Experiments were carried out at room temperature on nitrogen purged solution of anhydrous acetonitrile (AcN) containing TEAP $0.1 \mathrm{molL}^{-1}$ as supporting electrolyte, substrate $5 \times 10^{-4} \mathrm{molL}^{-1}$ and increasing amounts of 2,6-lutidine as deprotonating agent or water as protonating agent.

\subsubsection{Controlled potential electrolysis in UV-vis cell}

Controlled potential electrolyses were carried out with a potentiostat AMEL 552 coupled with an integrator AMEL 731 and a x/ y recorder LINSEIS L250E, using a three-electrode UV-vis cell modified for spectroelectrochemistry, and an Agilent 8453 diode array spectrophotometer. A platinum wire was used as working electrode, an $\mathrm{Ag}^{-\mathrm{AgClO}_{4}}\left(0.1 \mathrm{~mol} \mathrm{~L}^{-1}\right) / \mathrm{AcN}$-fine porosity fritted glass disk-AcN/Na$\mathrm{ClO}_{4}\left(0.1 \mathrm{molL}^{-1}\right)$-sintered glass disk as reference and a platinum wire as auxiliary electrode (placed on the inner wall of a glass tube containing AcN/TEAP $0.1 \mathrm{molL}^{-1}$ and connected to the solution via a sintered glass-disk). Experiments were carried out at room temperature on solution of anhydrous AcN containing TEAP $0.1 \mathrm{~mol} \mathrm{~L}^{-1}$ and CAF $7 \times 10^{-5} \mathrm{molL}^{-1}$ or TPh $1 \times 10^{-4} \mathrm{molL}^{-1}$, in turn. The electrolysis solutions were stirred by purging with a continuous nitrogen flux passing through a bubbler containing AcN to limit evaporation during prolonged experiments. Spectra were recorded at short time intervals during electrolysis.

\subsubsection{Chemical reactivity towards ROS: UV-vis experiments}

UV-vis experiments were carried out with an Agilent 8453 diode array spectrophotometer. Chemical reactivity of CAF, TBr and TPh was studied in anhydrous AcN, purged with nitrogen, by adding a) $\mathrm{PbO}_{2}$, b) $\mathrm{KO}_{2}$ and c) $\mathrm{G}$, in turn, directly in the UV-vis cell, according to the following procedures: a) small amounts of $\mathrm{PbO}_{2}$ were added to the starting solution containing $1 \times 10^{-4} \mathrm{molL}^{-1}$ of $\mathrm{CAF}, \mathrm{TBr}$ or $\mathrm{TPh}$, and 
then spectra were recorded every $30 \mathrm{~s}$ for $5 \mathrm{~min}$; b) an amount of ${\mathrm{a} \mathrm{KO}_{2}}$ solution, prepared as described above, was added to the starting solution containing $\mathrm{CAF}, \mathrm{TBr}$ or $\mathrm{TPh}$ until to obtain the same final concentration of $1 \times 10^{-4} \mathrm{molL}^{-1}$ for tested substrate and superoxide, and then spectra were recorded every $30 \mathrm{~s}$ for $5 \mathrm{~min}$; c) an amount of solution containing in turn $\mathrm{CAF}, \mathrm{TBr}$ or $\mathrm{TPh}$ was added to the starting solution containing $\mathrm{G}$ until to obtain the same final concentration of $1 \times 10^{-4} \mathrm{molL}^{-1}$ for tested substrate and galvinoxyl, and then spectra were recorded every $30 \mathrm{~s}$ for the first $5 \mathrm{~min}$ and every $15 \mathrm{~min}$ for the following $60 \mathrm{~min}$. The characteristic absorption of $\mathrm{G}$ at $\lambda=859 \mathrm{~nm}$, in a spectral range free from interferences, was monitored to evidence possible variation.

\subsubsection{DFT calculation}

CAF and TPh have been studied in the context of ab-initio total energy calculations based on the Density Functional Theory [23,24]. To this aim, the two molecules have been simulated in cubic supercells (size of 30 Ang.) with periodic boundary conditions using a plane wave basis sets. Norm conserving Troullier-Martins pseudopotentials [25] have been employed with an energy cutoff of 90 Ry for the plane waves and 360 Ry for the electron density. Geometric optimizations have been performed using the Perdew-Burke-Ernzerhof (PBE) [26] generalized gradient approximation for the exchange-correlation energy functional. After geometric optimization, the electronic structure and properties have been studied using the HSE hybrid functional [27] with $25 \%$ of exact exchange. In all the cases, Makov-Payne correction to the total energy has been applied [28]. The calculations have been performed using the Quantum Espresso suite [29].

\section{Results}

\subsection{Cyclic voltammetry}

Cyclic voltammograms of CAF and TBr (both $\mathrm{N}^{7}$-methyl substituted xanthines) appeared almost identical and characterized by a well-defined mono-electronic anodic peak (by comparison with ferrocene under the same experimental conditions $[30,31])$ at the same anodic potential value $\mathrm{E}_{\mathrm{ap}}$ of $+1.26 \mathrm{~V}$ vs $\mathrm{Ag} / \mathrm{AgClO}_{4}$; a corresponding well-defined cathodic wave was observed in the reverse scan, at the cathodic potential values $\mathrm{E}_{\mathrm{cp}}$ of $+1.14 \mathrm{~V}$ and $+1.15 \mathrm{~V}$, respectively (see Table 1 ). Cyclic voltammogram of CAF was shown in Fig. 1 (solid line).

Electrochemical data of $\mathrm{CAF}$ and $\mathrm{TBr}$ were analyzed according to the diagnostic tests reported in literature [32] and evidenced for both substrates a quasi-reversible anodic process diffusion controlled (based on the linear relationship of the anodic current intensity $\mathrm{I}_{\text {ap }}$ versus the square root of scan rate $\nu^{1 / 2}, R=0.9949$ for $C A F$ and $R=0.9999$ for $\mathrm{TBr}$ ). Diagnostic tests were consistent with a chemical reaction following the primary oxidation process, supporting for CAF and $\mathrm{TBr}$ an EC mechanism. The chemical reaction rate constants for these EC systems were estimated from the working curve [33] of the cathodic/anodic current ratio $I_{c p} / I_{a p}$ as a function of $\log k \tau$, where $\tau$ is the time required to cross the potential range from the half wave potential to the switching potential; the $\mathrm{I}_{\mathrm{cp}} / \mathrm{I}_{\mathrm{ap}}$ experimental values found for CAF (0.32, scan rate $\left.0.200 \mathrm{~V} \mathrm{~s}^{-1}\right)$ and $\mathrm{TBr}\left(0.27\right.$, scan rate $\left.0.200 \mathrm{Vs}^{-1}\right)$ lied in the linearity range of the working curve and values of $\mathrm{k} \cong 7 \mathrm{~s}^{-1}$ for $\mathrm{CAF}$ and $\mathrm{k} \cong 42 \mathrm{~s}^{-1}$ for $\mathrm{TBr}$ were estimated.

The electrochemical behavior of the $\mathrm{N}^{7}$-unsubstituted TPh appeared different from that one of CAF and TBr. Cyclic voltammogram of TPh under the same experimental conditions (shown in Fig. 2, solid line) was characterized by a slightly broadened anodic peak at the less positive $\mathrm{E}_{\mathrm{ap}}$ of $+1.22 \mathrm{~V}$ vs $\mathrm{Ag} / \mathrm{AgClO}_{4}$ (see Table 1 ), and no cathodic peak was observed in the reverse scan. The process involved slightly less than one-electron by comparison of the $I_{a p}$ value with that one of the mono-electronic system of ferrocene under the same experimental conditions [30,31]. Electrochemical data, analyzed according to the diagnostic tests [32], were consistent with an irreversible anodic process diffusion controlled (based on the linear relationship of $I_{\text {ap }}$ versus $\nu^{1 / 2}$, $\mathrm{R}=0.9981$ ). Because of the irreversibility of the anodic process, no kinetic data related to a possible following chemical reaction could be discussed on the base of cyclic voltammetry.

The effect of a deprotonating agent was checked on both CAF and TPh by adding increasing amounts of 2,6-lutidine to the tested solutions. No effect was observed for CAF, as predictable because of the lack of hydrogen atoms available for a proton transfer. On the contrary, the anodic peak of TPh decreased of about $14 \%$ when an equimolar amount of 2,6-lutidine was added to the tested solution and a second broadened anodic peak was observed at the less positive potential $+0.86 \mathrm{~V}$ (see Fig. 2 , dotted line).

The effect of a protonating agent was also checked on both CAF and TPh by adding increasing amounts of Milli-Q deionized water to the tested solutions. When an equimolar amount of water was added to the solution containing CAF, the cathodic peak in the reverse scan disappeared, the anodic peak was anodically shifted of $80 \mathrm{mV}$ $\left(\mathrm{E}_{\mathrm{ap}}{ }^{\mathrm{a}}=+1.34 \mathrm{~V}\right.$, Table 1$)$ and the anodic peak current increased till doubling, with $I_{a p}{ }^{a} / I_{a p}=2.2$ (Fig. 1, dotted line). When an equimolar amount of water was added to the solution containing $\mathrm{TPh}$, a similar behavior was observed, the anodic peak current increasing to $\mathrm{I}_{\mathrm{ap}}{ }^{\mathrm{a}} / \mathrm{I}_{\mathrm{ap}}=2.6$ and the anodic peak potential anodically shifting of $50 \mathrm{mV}$ $\left(\mathrm{E}_{\mathrm{ap}}{ }^{\mathrm{a}}=+1.27 \mathrm{~V}\right.$, Table 1$)$. The anodic potential shift had been previously observed for CAF in acidic medium [34], and might be likely ascribed to the protonating effect of water on one of the N-atoms, in the whole making harder the oxidation of the substrate.

All electrochemical data were resumed in Table 1.

\subsection{Controlled potential electrolysis in UV-vis cell}

The anhydrous AcN solution of CAF, characterized by absorptions at $\lambda / \mathrm{nm} 205$ and 274 and by a weak band in the region $220-230 \mathrm{~nm}$ (Fig. 3a, 1), was electrolyzed at a working platinum electrode at the experimental value $\mathrm{E}_{\mathrm{ap}}$ of $+1.20 \mathrm{~V}$, and spectra were recorded at short time intervals. Electrolysis was stopped when the current reached a constant plateau, in correspondence of which no further spectral changes were observed, with a total current consumption corresponding to 1.5 electrons per mole of substrate. After the consumption

Table 1

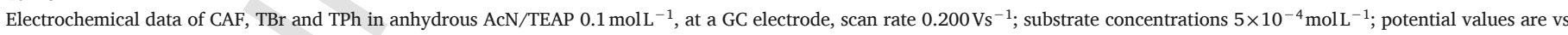
$\mathrm{Ag} / \mathrm{AgClO}_{4}$.

\begin{tabular}{|c|c|c|c|c|c|c|}
\hline$[\mathrm{S}]$ & $\mathrm{E}_{\mathrm{ap}}(\mathrm{V})$ & $\mathrm{E}_{\mathrm{cp}}(\mathrm{V})$ & $\mathrm{E}_{\mathrm{ap}}^{\mathrm{a}}(\mathrm{V})$ & $\mathrm{E}_{\mathrm{cp}}^{\mathrm{a}}(\mathrm{V})$ & $\mathrm{E}_{\mathrm{ap}}^{\mathrm{b}}(\mathrm{V})$ & $\mathrm{E}_{\mathrm{cp}}^{\mathrm{b}}(\mathrm{V})$ \\
\hline CAF & +1.26 & +1.14 & +1.34 & - & +1.26 & +1.14 \\
\hline $\mathrm{TBr}$ & +1.26 & +1.15 & Not measured & Not measured & Not measured & Not measured \\
\hline $\mathrm{TPh}$ & +1.22 & - & +1.27 & - & $+0.86 ;+1.22$ & - \\
\hline
\end{tabular}

a In the presence of an equimolar amount of water.

b In the presence of an equimolar amount of 2,6-lutidine. 


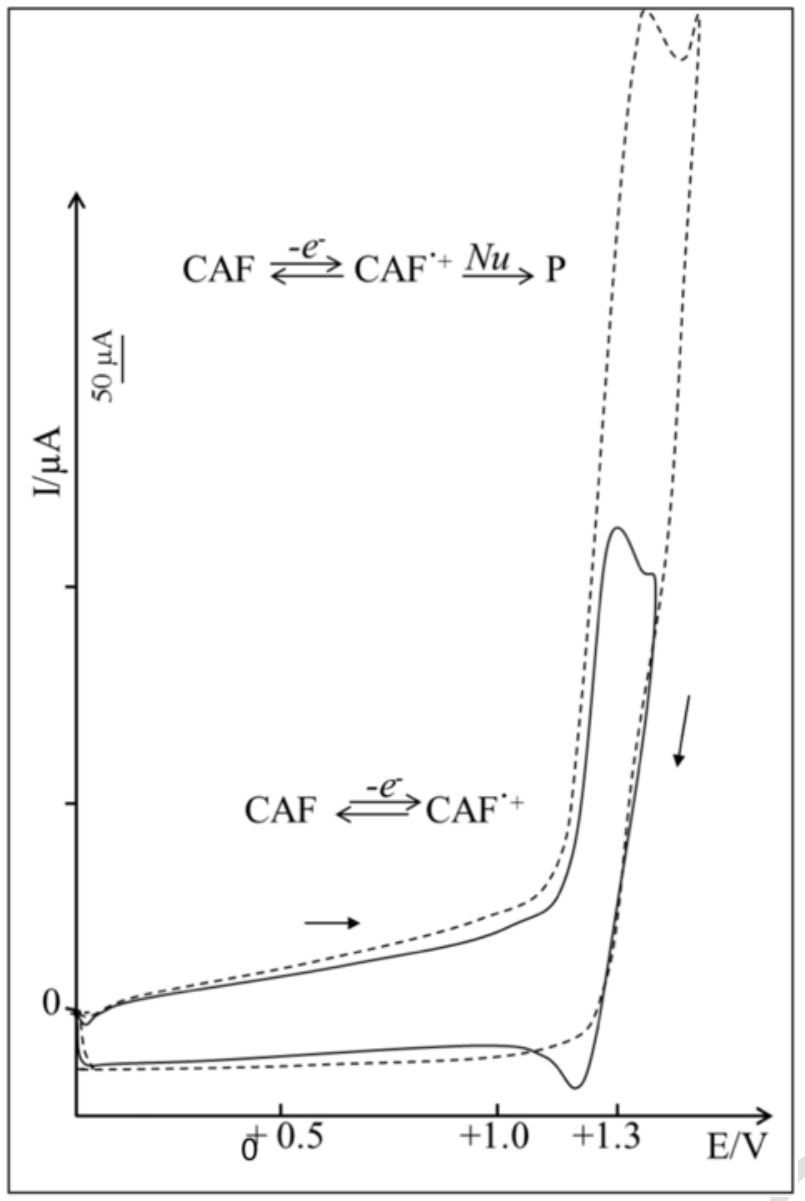

Fig. 1. Cyclic voltammetry of CAF in anhydrous AcN/TEAP $0.1 \mathrm{molL}^{-1}$, at GC electrode, vs $\mathrm{Ag} / \mathrm{AgClO}_{4}$, scan rate $0.200 \mathrm{Vs}^{-1}$, in the absence (solid line) and in the presence (dotted line) of an equimolar amount of water.

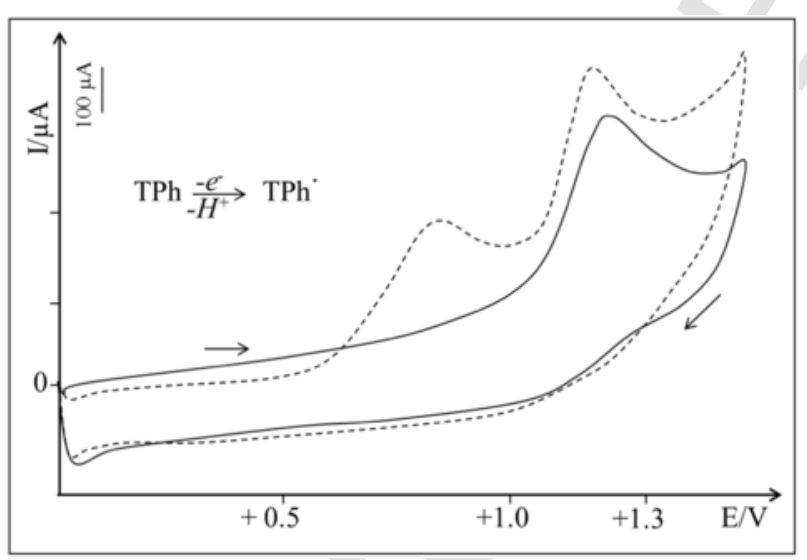

Fig. 2. Cyclic voltammetry of TPh in anhydrous AcN/TEAP $0.1 \mathrm{molL}^{-1}$, at GC electrode, vs $\mathrm{Ag} / \mathrm{AgClO}_{4}$, scan rate $0.200 \mathrm{Vs}^{-1}$, in the absence (solid line) and in the presence (dotted line) of 2,6-lutidine as deprotonating agent.

of an amount of current consistent with the theoretical value for a mono-electronic process, a strong decrease of absorbance at both $205 \mathrm{~nm}$ and $274 \mathrm{~nm}$ and the increase of a new weak absorption in the region 350-410 nm (Fig. 3a, 2) were observed. The UV-vis spectrum of the final electrolyzed solution was characterized by a red-shift of $205 \mathrm{~nm}$ to $216 \mathrm{~nm}$, a blue-shift of $274 \mathrm{~nm}$ to $266 \mathrm{~nm}$, the loss of the weak band at $220-230 \mathrm{~nm}$, and a further increase of the new band at
350-410 nm (Fig. 3a, 3). This final spectrum remained unchanged for at least $15 \mathrm{~min}$ after electrolysis had been stopped.

The starting solution of TPh showed the characteristic absorption at $\lambda(\mathrm{AcN}) / \mathrm{nm} 201$ and 272, slightly blue shifted with respect to CAF, in agreement with the calculated values reported below $(265 \mathrm{~nm})$, and a weak band in the region of $220-230 \mathrm{~nm}$ (Fig. 3b, 1). Electrolysis was carried out at the experimental value $\mathrm{E}_{\mathrm{ap}}$ of $+1.20 \mathrm{~V}$ and a different trend with respect to CAF was observed. Electrolysis was stopped when the current reached a constant plateau, in correspondence of which no further spectral changes were observed, with a total current consumption consistent with a mono-electronic process. The UV-vis spectrum of the final electrolyzed solution (Fig. 3b, 2) was characterized by a decrease of the absorption at $201 \mathrm{~nm}$ and a very small decrease of the absorption at $272 \mathrm{~nm}$, with a $2 \mathrm{~nm}$ blue-shift $(270 \mathrm{~nm})$. The weak band in the region $220-230 \mathrm{~nm}$ was still present.

\subsection{DFT calculations}

A computational analyses based on the DFT was performed on CAF and $\mathrm{TPh}$ molecular structure: the ground state configurations of CAF and TPh were shown in Fig. 4, and the relevant bond distances and angles of both studied compounds were reported in Table S1.

The geometric configurations of CAF and TPh resulted quite similar concerning the hexa-cyclic moiety, while some differences regarding mainly the angles were found for the penta-cyclic moiety: the distortions particularly affected the angles with $\mathrm{N}^{7}$ and $\mathrm{C}^{8}$ at the vertices. The density of states (DOS) involving the last occupied molecular orbital (MO) of CAF and TPh were shown in Fig. 5, where the reference zero energy is the HOMO energy; the HOMO-1 and HOMO-2 molecular orbitals of TPh appeared shifted upwards with respect to those ones of CAF by nearly $50 \mathrm{meV}$ (Fig. 5).

The projected density of states (PDOS) of the HOMO orbital was shown in Fig. 6 and indicated for CAF (Fig. 6a) a large contribute due to the $\mathrm{C}^{4}-\mathrm{C}^{5}$ bond, i.e. the bond shared by the hexa-cyclic and penta-cyclic moieties, besides contributes due to the oxygen atoms $\mathrm{O}^{13}$ and $\mathrm{O}^{11}$ and, at a lesser extent, to $\mathrm{C}^{6}$ and $\mathrm{C}^{2}$.

The HOMO-LUMO energy difference was measured and resulted in nearly $4.6538 \mathrm{eV}$ for $\mathrm{CAF}$, corresponding to an absorption peak at $\lambda$ $266 \mathrm{~nm}$, close to the experimental value of $274 \mathrm{~nm}$ reported above.

The PDOS analysis of TPh (Fig. 6b) showed different features especially concerning the HOMO, that in this case contained also large contributes from both $\mathrm{N}^{7}$ and $\mathrm{C}^{8}$ of the imidazole group, besides being shared by the oxygen atoms and the $\mathrm{C}^{4}-\mathrm{C}^{5}$ bond similarly to CAF.

Also in this case, the HOMO-LUMO energy difference was measured and corresponded to an adsorption peak at $265 \mathrm{~nm}$, rather close to the experimental value of $272 \mathrm{~nm}$ reported above.

The PDOS HOMO decomposition differences between CAF and TPh evidenced that, excluding the $\mathrm{O}$ atoms, the lowest energy electrons were spread on the $\mathrm{C}$ atoms with some prevalence on the hexa-cyclic moiety in the case of CAF, while in the case of TPh the lowest energy electrons state was shared by $\mathrm{C}^{4}-\mathrm{C}^{5}$ atoms and the $\mathrm{N}$ atoms of both moieties, with a large contribute from the imidazole penta-cyclic moiety.

\subsection{Chemical reactivity towards ROS: UV-vis experiments}

Chemical reactions of CAF, TBr and TPh towards $\mathrm{PbO}_{2}, \mathrm{O}_{2}{ }^{*-}$ and G were carried out in UV-vis cell and monitored recording spectra at short time intervals, as described above. In all the cases, no spectral change was observed, as evidenced in Fig. 7 where the behavior of CAF towards $\mathrm{PbO}_{2}, \mathrm{O}_{2}{ }^{--}$and $\mathrm{G}$ was reported, as an example. 


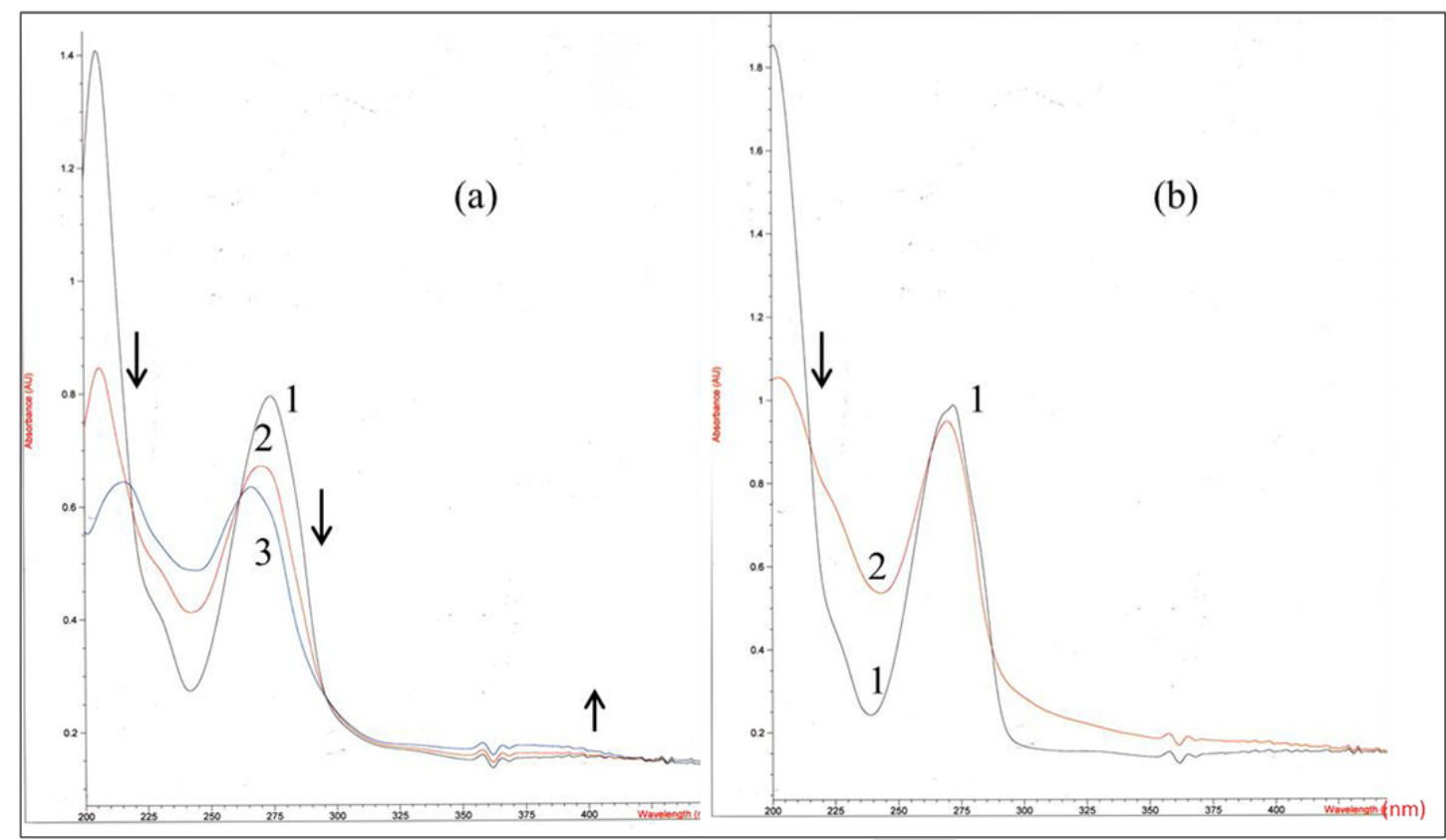

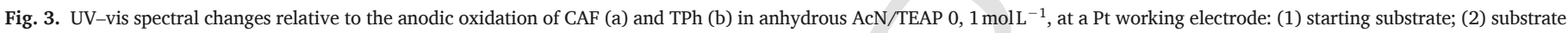
after the mono-electronic oxidation; (3) final oxidized solution of CAF.
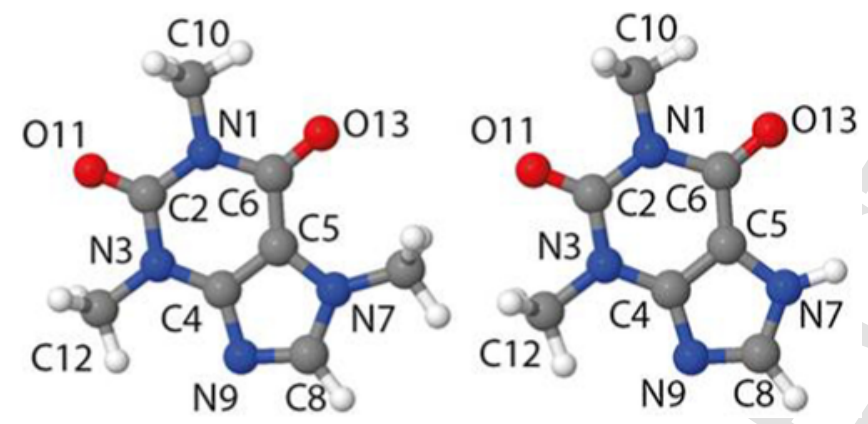

Fig. 4. CAF (left) and TPh (right) ground state configurations.

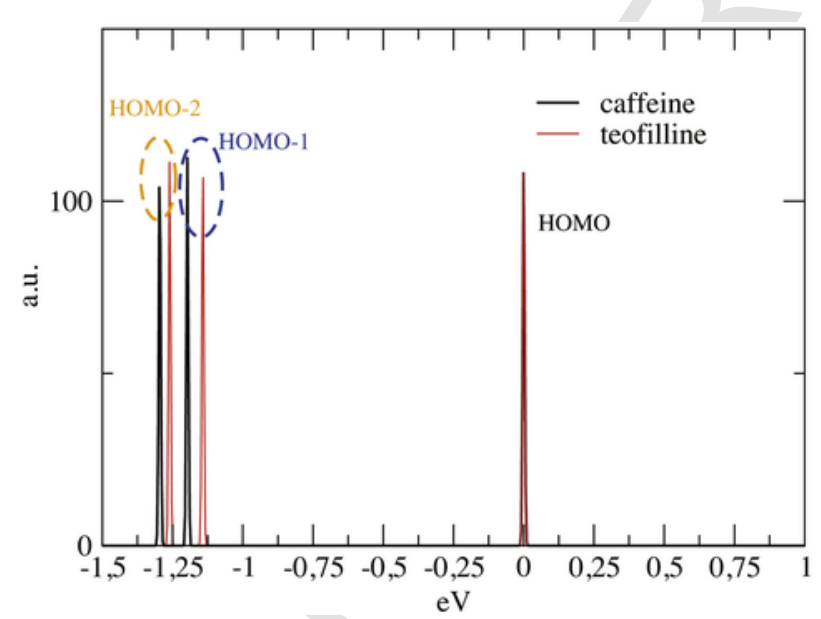

Fig. 5. CAF and TPh density of states (DOS) of the highest occupied MO states.

\section{Discussion}

A new insight into the redox properties of CAF was obtained combining theoretical calculations on $\mathrm{CAF}$ and $\mathrm{TPh}$ molecular structures with the comparative electrochemical study of $\mathrm{CAF}, \mathrm{TBr}$ and $\mathrm{TPh}$, and the chemical study of interactions of CAF, TBr and TPh with ROS mimics, in aprotic medium favorable to radical intermediates.

The electrochemical study evidenced strong similarities between $\mathrm{CAF}$ and $\mathrm{TBr}$, suggesting that $\mathrm{N}^{1}$ atom (see Fig. 4) could not be involved in the oxidation mechanism, while a different behavior was found for $\mathrm{TPh}$, suggesting that different oxidative pathways might occur for $\mathrm{N}^{7}$-substituted and $\mathrm{N}^{7}$-unsubstituted methylxanthines.

First, cyclic voltammetry evidenced a quasi-reversible mono-electronic anodic oxidation for $\mathrm{CAF}$ and $\mathrm{TBr}$, and an irreversible mono-electronic anodic oxidation for TPh (Section 3.1).

Second, an identical $\mathrm{E}_{\mathrm{ap}}$ value was measured for $\mathrm{CAF}$ and $\mathrm{TBr}$ $\left(+1.26 \mathrm{~V}\right.$, vs $\left.\mathrm{Ag} / \mathrm{AgClO}_{4}\right)$ while a less positive $\mathrm{E}_{\mathrm{ap}}$ value was measured for $\mathrm{TPh}\left(+1.22 \mathrm{~V}\right.$, vs $\left.\mathrm{Ag} / \mathrm{AgClO}_{4}\right)$. The same trend had been previously observed in aqueous medium [18], and had sounded unexpected already at that time. In fact, an opposite trend could be predicted on the bases of the stabilizing effect due to the $\mathrm{N}^{7}$-methylation of CAF and $\mathrm{TBr}$ with respect to $\mathrm{TPh}$, especially if the double bond $\mathrm{C}^{8}-\mathrm{N}^{9}$ (Fig. 4) was supposed to be involved in the primary oxidation process as mainly reported in literature. According to our knowledge, no interpretation of this behavior has been proposed up to now.

Some differences regarding the penta-cyclic moiety were also evidenced by computational analysis of CAF and TPh molecular structure: a) the angles distortion, particularly affecting the angles with $\mathrm{N}^{7}$ and $\mathrm{C}^{8}$ at the vertices (Table S1), b) the density of states (DOS) of the last occupied MO, with HOMO-1 and HOMO-2 of TPh shifted of $50 \mathrm{meV}$ upwards with respect to CAF (Fig. 5), and above all c) the PDOS HOMO decomposition (Fig. 6), evidencing that the lowest energy electrons of CAF were spread on the hexa-cyclic moiety with a large contribute from the $C^{4}-C^{5}$ bond, while the lowest energy electrons of TPh were shared with the penta-cyclic moiety with a large contribute from both $\mathrm{N}^{7}$ and $C^{8}$ atoms (Section 3.3). This analysis supported the electrochemical data suggesting that different oxidative pathways might occur for CAF (and likely $\mathrm{TBr}$ ) and $\mathrm{TPh}$.

Calculations and experimental data of the present study were consistent with the mono-electronic oxidation of the $\mathrm{C}^{4}-\mathrm{C}^{5}$ double bond of $\mathrm{CAF}$ to the corresponding radical cation $\mathrm{CAF}^{\bullet+}$, stable enough to be reversibly reduced to the starting material (Fig. 1, solid line) or avail- 

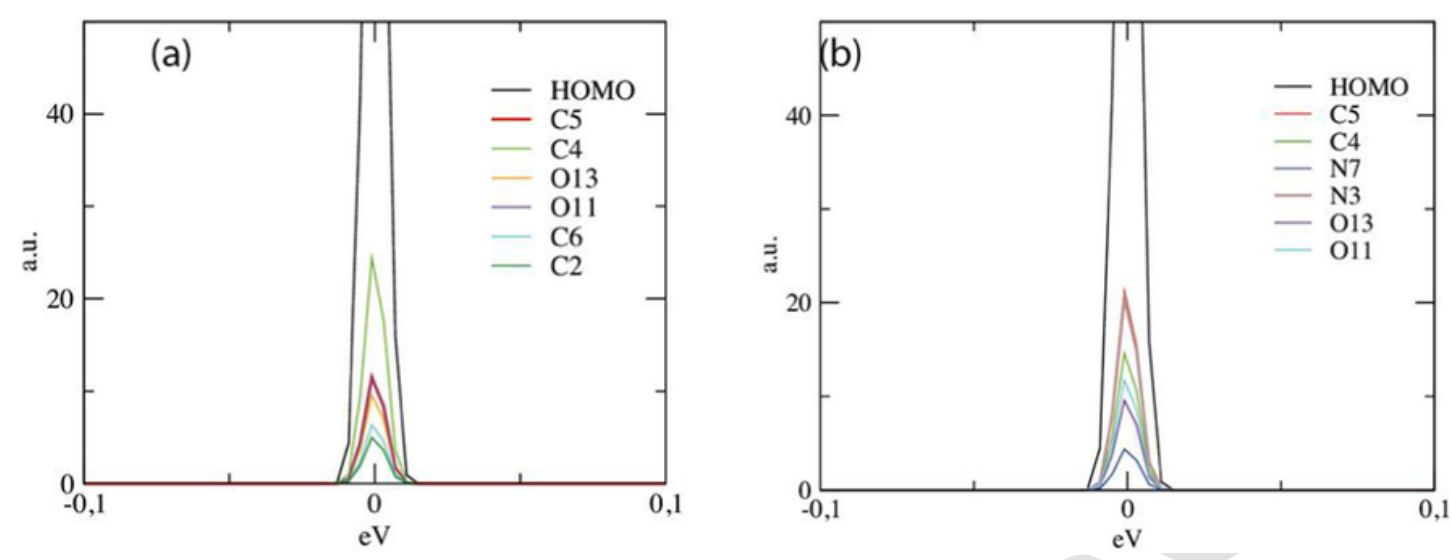

Fig. 6. Projected density of states (PDOS) HOMO decomposition of CAF (a) and TPh (b).

able to react with a nucleophilic substrate (water, as an example), according to an EC mechanism. In fact, the reversibility of the system $\mathrm{CAF} / \mathrm{CAF}^{*+}$ was removed by adding an amount of water equimolar with substrate, and the observed doubling of the anodic peak current (Section 3.1) (Fig. 1, dotted line) might be explained with a following chemical reaction (EC mechanism) whose product could be furtherly oxidized at the same potential value. A pattern for the reactivity of the electro-generated $\mathrm{CAF}^{*+}$ in the presence of water as a nucleophilic reagent was shown in Scheme 1.

Calculations and experimental data of $\mathrm{TPh}$ were instead consistent with an irreversible mono-electronic oxidation coupled with a consecutive proton transfer, likely from $\mathrm{N}^{7}$, to give the corresponding neutral radical $\mathrm{TPh}^{*}$. The involvement of a proton transfer was supported by the effect of a deprotonating agent added to the TPh solution: in fact, the second anodic peak at the less positive potential $+0.86 \mathrm{~V}$ evidenced in the presence of an equimolar amount of 2,6-lutidine (Fig. 2, dotted line) might be likely ascribed to the easier oxidation of the imidazolium anion of TPh to the corresponding neutral radical. The broadened shape of this anodic peak at $+0.86 \mathrm{~V}$ was also in agreement with the involvement of an acid-base equilibrium, the $\mathrm{pK}_{\mathrm{b}}$ value of 2,6-lutidine and the $\mathrm{pK}_{\mathrm{a}}$ value of imidazole (chosen as a reference value for $-\mathrm{N}^{7} \mathrm{H}$ of $\mathrm{TPh}$ ) being 7.40 and 6.95 , respectively.

Noteworthy, the electrochemical analogies between CAF and $\mathrm{TBr}$ suggested that a neutral radical might be excluded for $\mathrm{TBr}$, in spite of the presence of a proton available on $\mathrm{N}^{1}$ atom, to further support the involvement of the $\mathrm{N}^{7}$ atom in the oxidation of TPh.

Spectral changes evidenced during controlled potential electrolysis of CAF and TPh (Section 3.2) were in agreement with these different oxidative paths leading to the radical cation $\mathrm{CAF}^{*+}$ and the neutral radical $\mathrm{TPh}^{\bullet}$, respectively. In fact, the UV-vis spectrum recorded after the selective mono-electronic anodic oxidation of CAF evidenced a strong decrease of absorptions at $205 \mathrm{~nm}$ and $274 \mathrm{~nm}$ (Fig. 3a, 2), in agreement with the loss of the basic conjugated structure of $\mathrm{CAF}^{\bullet+}$ (Scheme $1, \mathrm{I})$. On the contrary, the UV-vis spectrum recorded after the selective mono-electronic anodic oxidation of TPh evidenced a decreased absorbance at $201 \mathrm{~nm}$ but a very slight decrease of absorption at $272 \mathrm{~nm}$ (Fig. 3b, 2). This was in agreement with a conjugated structure mainly preserved, as expected for the neutral radical TPh ${ }^{*}$ generated by fast deprotonation of the electro-generated radical cation (Scheme 2, II).

Spectra 2 in Fig. 3a and b recorded after the mono-electronic anodic oxidation of CAF and $\mathrm{TPh}$, respectively, might be assigned as the UV-vis spectra of the radical cation $\mathrm{CAF}^{*+}$ and the neutral radical $\mathrm{TPh}^{*}$, respectively.

Also UV-vis data of full electrolysis of CAF and TPh seemed to support the nature of their first oxidation products. As discussed above, the electro-generated radical cation $\mathrm{CAF}^{\bullet+}$ can be considered an easy substrate for nucleophiles; so the total current consumption, higher than that one expected for a mono-electronic process, might be due to the oxidation, at the same operative potential, of the product of a following chemical reaction (see Scheme 1). In fact, despite the use of anhydrous solvent, the presence of water in trace in the electrolyzed solution could not be ruled out, mainly because of the prolonged purging with nitrogen.

The same might occur to the neutral radical $\mathrm{TPh}^{\bullet}$ (as evidenced by cyclic voltammetry in the presence of stoichiometric amount of water, discussed above), but the electrolysis contest might be more favorable to a coupling reaction because of the high amount of $\mathrm{TPh}^{*}$ produced, and in that case no further oxidation at the same potential should occur.

The reaction pattern proposed for the reactivity of $\mathrm{CAF}^{\bullet+}$, resumed in Scheme 1, seemed also in agreement with the involvement of the 4,5-diole derivative of CAF postulated in literature to explain some degradation products of CAF detected by mass spectrometry [16]. Similarly, the reaction pattern proposed for the reactivity of $\mathrm{TPh}^{*}$, resumed in Scheme 2, seemed also in agreement with the formation of a dimeric form of TPh under anodic oxidation reported in literature [35].

The reactivity of CAF towards ROS, here briefly discussed, was also evaluated by reacting CAF with: (a) $\mathrm{PBO}_{2}$, an oxidant mainly working as H-atom acceptor [30], (b) $\mathrm{O}_{2}{ }^{*-}$, a quite important ROS considered responsible of oxidative stress, whose complex reactivity includes ET and HT mechanisms as well as proton-induced disproportionation leading to strong oxidants [36], and (c) the O-centered stable free radical G, a model for ROS and a different reactant with respect to the N-centered stable radical DPPH towards which CAF had been reported inactive in literature [11].

No spectral changes were observed for CAF in all the cases (Section 3.4, Fig. 7), suggesting that antiradical activity, that is the capacity to react with free radicals in a single reaction [13], can hardly be predicted for CAF. This data suggested that no H-atoms of CAF, the methyl ones as well as that one on $\mathrm{C}^{8}$, can be considered available for an $\mathrm{H}$-atom transfer (HT) mechanism.

Results towards $\mathrm{O}_{2}{ }^{--}$were also in agreement with literature [12]; on the other hand, an ET mechanism between CAF and superoxide could be excluded on the base of the respective electrochemical potential values, the reduction potential of superoxide being too negative [20] to oxidize CAF. Also a proton transfer from CAF to $\mathrm{O}_{2}{ }^{--}$might be hardly predicted, on the base of the behavior of CAF in the presence of a base (described above), that means no proton-induced disproportionation of superoxide should be expected, at least due to CAF, to produce other ROS.

Also in the case of $\mathrm{TBr}$ and $\mathrm{TPh}$, with other $\mathrm{H}$-atoms available on $\mathrm{N}^{1}$ and $\mathrm{N}^{7}$, respectively, no reactivity was observed, furtherly to support 


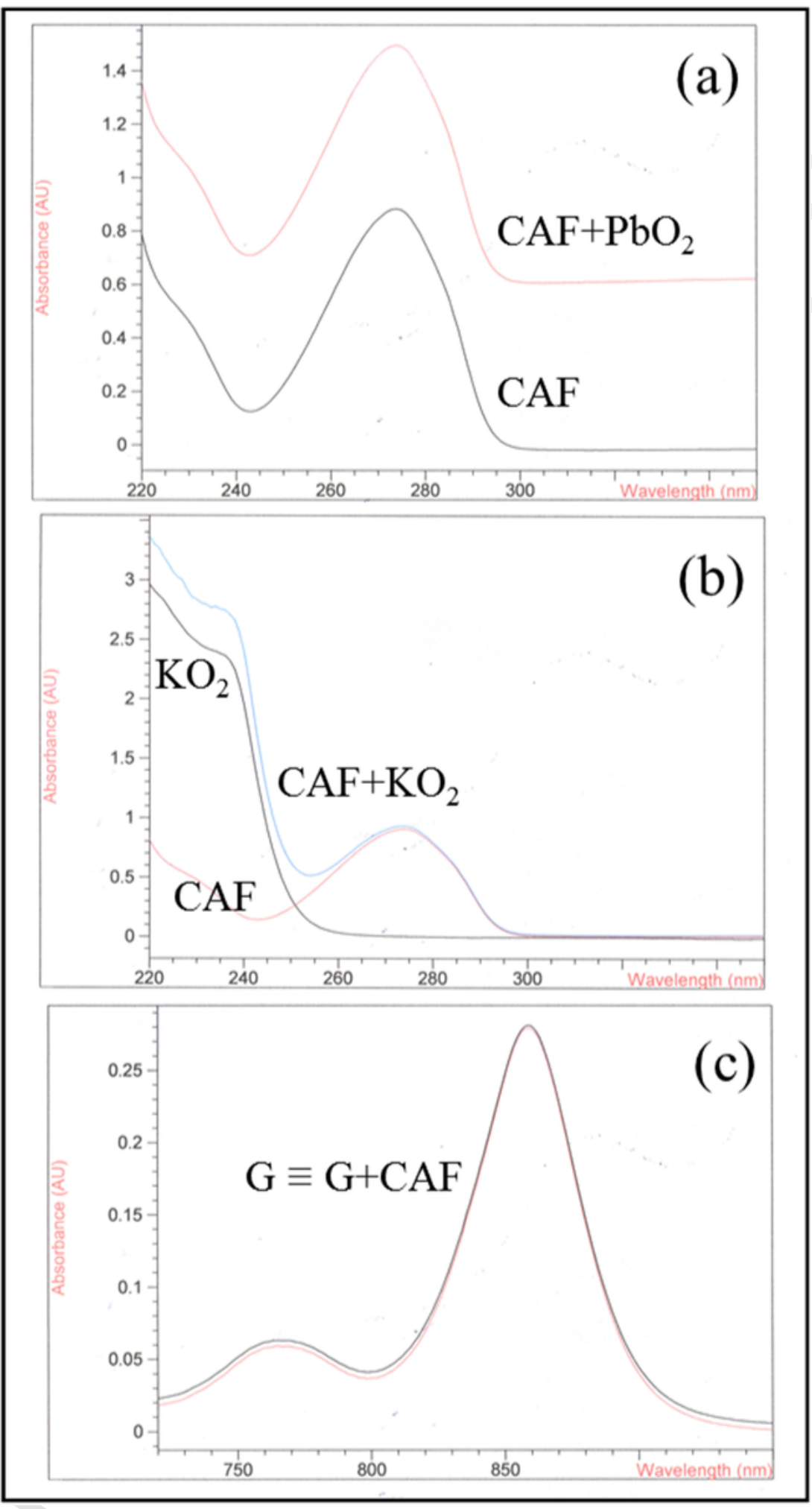

Fig. 7. UV-vis spectral changes relative to the chemical reaction of $\mathrm{CAF}$ with (a) $\mathrm{PbO}_{2}$, (b) $\mathrm{KO}_{2}$ and (c) G, in anhydrous AcN.

that antiradical properties should be ruled out for these methylxanthines.

In the light of the results of the present study, the acclaimed scavenging activity of CAF towards $\mathrm{HO}^{*}$ should be reviewed; the reported reactivity should be better interpreted in terms of an ET mechanism and based on the strong oxidative power of $\mathrm{HO}^{*}$ rather than on pre- sumed antioxidant properties of CAF. In fact, with its reduction potential value, highly positive, of $+2.72 \mathrm{~V},+2.31 \mathrm{~V}$ and $+1,77 \mathrm{~V}$ (vs NHE) at $\mathrm{pH}$ values 0,7 and 14 , respectively [36], $\mathrm{HO}^{*}$ can react thermodynamically with most of the reductant substrates, also the weakest. CAF can be considered a very weak reductant because of its highly positive potential of $+1.26 \mathrm{~V}$ vs $\mathrm{Ag} / \mathrm{Ag}^{+}(+1.64 \mathrm{~V}$ vs SCE, measured 


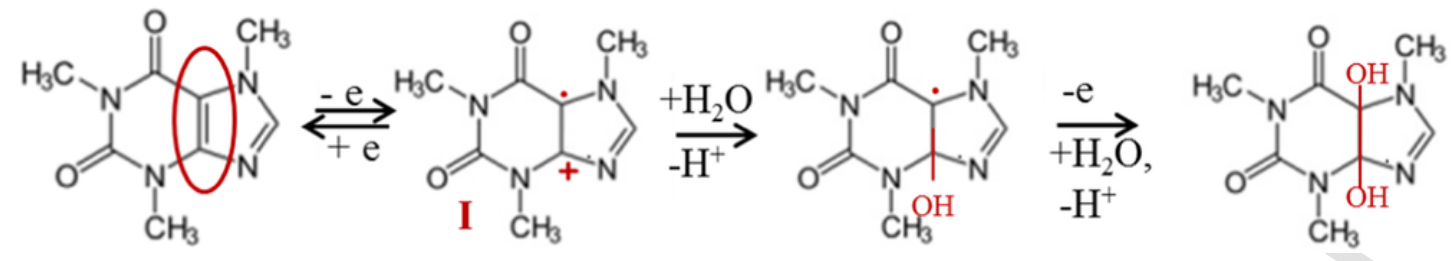

Scheme 1. Mono-electronic anodic oxidation of CAF and a following reaction pattern proposed for the radical cation CAF ${ }^{\bullet}$ (I) in the presence of water as a nucleophile.

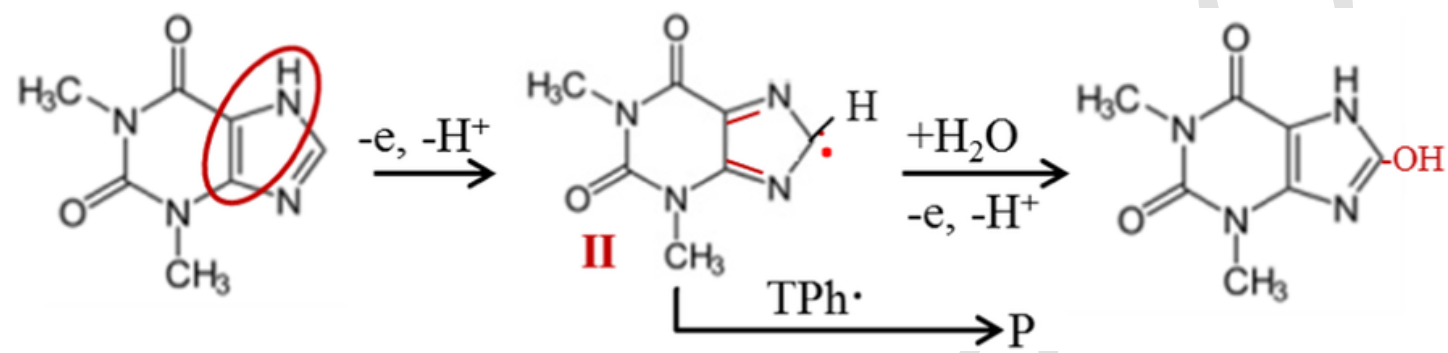

Scheme 2. Mono-electronic anodic oxidation of TPh and a pattern proposed for possible competitive reactions of the neutral radical TPh ${ }^{\bullet}$ (II).

value, $+1.89 \mathrm{~V}$ vs NHE, calculated value) but can be thermodynamically oxidized by $\mathrm{HO}^{*}$, at least at $\mathrm{pH}$ from acidic to neutral $(+2.72 \mathrm{~V}$ and $+2,31 \mathrm{~V}$, respectively, vs NHE). In highly alkaline solution, the most similar to a lipophilic environment, the anodic potential value of $\mathrm{HO}^{*}(+1.77 \mathrm{~V}$ at $\mathrm{pH}=14$, vs NHE $)$ appeared even less positive than CAF $(+1.89 \mathrm{~V}$ in aprotic medium, vs NHE), but an ET might be still predicted on the base of the Marcus theory, according to which an ET reaction may occur even when the $\mathrm{E}_{\mathrm{cp}}$ (oxidant) is less positive than the $\mathrm{E}_{\mathrm{ap}}$ (reductant) with a difference not bigger than $0.4 \mathrm{~V}$ [37]. So CAF was expected to react with $\mathrm{HO}^{*}$ as the most of reductant substrates. An analogous behavior might be predicted for $\mathrm{TBr}$ and $\mathrm{TPh}$.

A possible reaction pattern for the oxidation of CAF as well as of TPh by $\mathrm{HO}^{*}$ has been proposed in Scheme $3 \mathrm{a}$ and b, respectively.

\section{Conclusions}

The present work arose from the need to clear up the role of CAF as a potential antioxidant. In fact, a hydroxyl free radical scavenging ability has been recently acclaimed for CAF and a potential antioxidant capacity towards ROS proposed as a consequence. A new insight into the oxidative mechanism of CAF was obtained by using as combined tools cyclic voltammetry, controlled potential electrolyses carried out in UV-vis cell, DFT calculations and an investigation on the chemical reactivity towards ROS mimics, carried out by UV-vis spectrophotometry. All the experiments were carried out in a medium poor of proton, closer to a lipophilic environment and more favorable to radical intermediates. The study was extended to $\mathrm{TBr}, \mathrm{N}^{7}$-substituted as CAF, and to the $\mathrm{N}^{7}$-unsubstituted TPh.

All data were consistent with the primary oxidation of the $C^{4}-C^{5}$ double bond of the studied methylxanthines, according to an ET process leading to the corresponding quite stable radical cation for the $\mathrm{N}^{7}$-substituted $\mathrm{CAF}$ and $\mathrm{TBr}$, and an ET process coupled to a consecutive proton transfer and leading to the corresponding neutral radical for the $\mathrm{N}^{7}$-unsubstituted TPh. A different following reactivity was then predicted for the radical cation $\mathrm{CAF}^{\bullet+}$ (and likely $\mathrm{TBr}^{*}$ ) ) and the neutral radical TPh -, due to their own different nature. 4,5-Saturated derivatives might be expected from the oxidation of $\mathrm{CAF}$ and 8-substituted derivatives might be expected for TPh.

No interaction was evidenced between CAF, TBr or TPh, and ROS as $\mathrm{PbO}_{2}$, superoxide radical anion and galvinoxyl free radical, suggesting

(a)

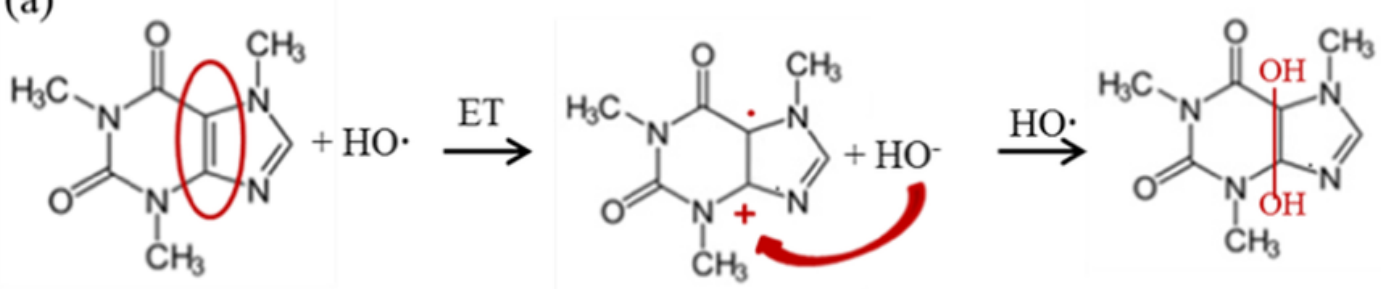

(b)<smiles>Cn1c(=O)c2cc3[nH]c([OH2+])nc3nc2n(C)c1=O</smiles>

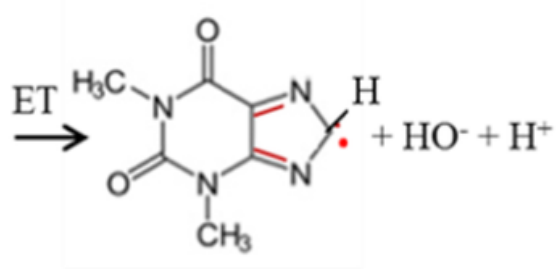<smiles>Cn1c(=O)c2[nH]c(O)nc2n(C)c1=O</smiles>

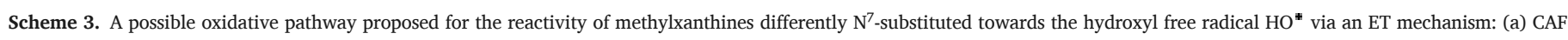
and (b) TPh. 
that antiradical properties should be excluded for these methylxanthines.

With regard to the antioxidant properties, the high oxidation potential, in aprotic medium and even higher in aqueous medium, makes CAF and related compounds hard to be oxidized, suggesting that these compounds can hardly be considered antioxidants, in general. The hydroxyl free radical scavenging activity ascribed to CAF should be interpreted in terms of ET mechanism, according to the reactivity of most of the reductants, also weak as methylxanthines, in the presence of strong oxidants as $\mathrm{HO}^{*}$.

Supplementary data to this article can be found online at https:// doi.org/10.1016/j.bbagen.2018.05.011.

\section{Transparency document}

The Transparency document associated with this article can be found, in online version.

\section{Acknowledgments}

We thank the Ministero dell'Università e della Ricerca Scientifica e Tecnologica for financial support. Rita Petrucci thanks Professor Giancarlo Marrosu, just retired, for more than 25 years of scientific support and sincere friendship.

\section{References}

[1] J. Monteiro, Marco G. Alves, Pedro F. Oliveira, Branca M. Silva, Pharmacological potential of methylxanthines: retrospective analysis and future expectations, Crit. Rev. Food Sci. Nutr. (2018) https://doi.org/10.1080/10408398.2018.1461607.

[2] M. Shechter, G. Shalmon, M. Scheinowitz, N. Koren-Morag, M.S. Feinberg, D. Harats, B.A. Sela, Y. Sharabi, P. Chouraqui, Impact of acute caffeine ingestion on endothelial function in subjects with and without coronary artery disease, Am. J. Cardiol. 107 (2011) 1255-1261.

[3] S.P. Gaytan, R. Pasaro, Neonatal caffeine treatment up-regulates adenosine receptors in brainstem and hypothalamic cardio-respiratory related nuclei of rat pups, Exp. Neurol. 237 (2012) 247-259.

[4] M.C. Wardle, M.T. Treadway, H. de Wit, Caffeine increases psychomotor perfor mance on the effort expenditure for rewards task, Pharmacol. Biochem. Behav. 102 (2012) 526-531.

[5] N. Ward, C. Whitney, D. Avery, D. Dunner, The analgesic effects of caffeine in headache, Pain 44 (1991) 151-155.

[6] B. Renner, G. Clarke, T. Grattan, A. Beisel, C. Muller, U. Werner, G. Kobal, K. Brune, Caffeine accelerates absorption and enhances the analgesic effect of acetaminophen, J. Clin. Pharmacol. 47 (2007) 715-726.

[7] T.P.A. Devasagayam, J.P. Kamat, H. Mohan, P.C. Kesavan, Caffeine as an antioxidant: inhibition of lipid peroxidation induced by reactive oxygen species, Biochim. Biophys. Acta 1282 (1996) 63-70.

[8] J.R.P. Prasanthi, B. Dasari, G. Marwarha, T. Larson, X. Chen, Caffeine protects against oxidative stress and Alzheimer's disease-like pathology in rabbit hippocampus induced by cholesterol-enriched diet, Free Radic. Biol. Med. 49 (2010) $1212-1220$.

[9] S.D. Varma, K.R. Hegde, Kynurenine-induced photo oxidative damage to lens in vitro: protective effect of caffeine, Mol. Cell. Biochem. 340 (2010) 49-54.

[10] R.H. Stadler, L.B. Fay, Antioxidative reactions of caffeine: formation of 8-oxocaffeine (1,3,7-trimethyluric acid) in coffee subjected to oxidative stress, J. Agric. Food Chem. 43 (1995) 1332-1338.

[11] V. Brezová, A. Šlebodová, A. Staško, Coffee as a source of antioxidants: an EPR study, Food Chem. 114 (2009) 859-868.

[12] J.R. León-Carmona, A. Galano, Is caffeine a good scavenger of oxygenated free rad icals?, J. Phys. Chem. 115 (2011) 4538-4546.

[13] G. Tirzitis, G. Bartosz, Determination of antiradical and antioxidant activity: basic principles and new insights, Acta Biochim. Polon. ABP, Review 57 (2010) 139-142.
[14] J.P. Telo, A.J.S.C. Vieira, Mechanism of free radical oxidation of caffeine in aqueous solution, J. Chem. Soc. Perkin Trans. 2 (1997) 1755-1757.

[15] M.R. Kumar, M. Adinarayana, Oxidation of caffeine by phosphate radical anion in aqueous solution under anoxic conditions, Proc. Indian Acad. Sci. (Chem. Sci.) 112 (2000) 551-557.

[16] I. Dalmázio, L.S. Santos, R.P. Lopes, M.N. Eberlin, R. Augusti, Advanced oxidation of caffeine in water: on-line and real-time monitoring by electrospray ionization mass spectrometry, Environ. Sci. Technol. 39 (2005) 5982-5988.

[17] B.H. Hansen, G. Dryhurst, Electrochemical oxidation of theobromine and caffeine at the pyrolitic graphite electrode, Electroanal. Chem. Interfacial Electrochem. 30 (1971) 407-416

[18] B.H. Hansen, G. Dryhurst, Voltammetric oxidation of some biologically importan xanthines at the pyrolitic graphite electrode, Electroanal. Chem. Interfacial Electrochem. 30 (1971) 417-426.

[19] K.K. Chan, R. Ganguly, Y. Li, R.D. Webster, Electrocehmically controlled one-elec trone oxidation coupled to consecutive hydrogen atom transfer of caffeine, ChemElectroChem 1 (2014) 1557-1562.

[20] R. Petrucci, E. Giorgini, E. Damiani, P. Carloni, G. Marrosu, A. Trazza, G.P. Littarru, L. Greci, Study on the interactions between coenzyme $Q_{0}$ and superoxide anion. Could ubiquinones mimics superoxide dismutase (SOD)?, Res. Chem. Intermed. 26 (2000) 269-282.

[21] R. Kuhn, I. Hammer, Active lead dioxide for the preparation of sensitive quinones, Chem. Ber. 83 (1950) 413-414.

[22] R. Andruzzi, A. Trazza, L. Greci, L. Marchetti, Electrochemical and ESR study on the reduction mechanism of some indoxyl derivatives in DMF, Ann. Chim. (Rome, Italy) 69 (1979) 583-596.

[23] P. Hohenberg, W. Kohn, Inhomogeneous electron gas, Phys. Rev. 136 (1964) B864

[24] W. Kohn, L.J. Sham, Self-consistent equations including exchange and correlation effects, Phys. Rev. 140 (1965), A1133.

[25] N. Troullier, J.L. Martins, Efficient pseudopotentials for plane-wave calculations, Phys. Rev. B 43 (1991) 1993-2006.

[26] J. Perdew, K. Burke, M. Ernzerhof, Generalized gradient approximation made simple, Phys. Rev. Lett. 77 (1996) 3865-3868.

[27] J. Heyd, G.E. Scuseria, M. Ernzerhof, Erratum: "Hybrid functionals based on a screened Coulomb potential", J. Chem. Phys. 124 (2006) 219906.

[28] G. Makov, M.C. Payne, Periodic boundary conditions in ab initio calculations, Phys. Rev. B 51 (1995) 4014-4022.

[29] P. Giannozzi, S. Baroni, N. Bonini, M. Calandra, R. Car, C. Cavazzoni, D. Ceresoli, G.L. Chiarotti, M. Cococcioni, I. Dabo, A. Dal Corso, S. de Giron- coli, S. Fabris, G. Fratesi, R. Gebauer, U. Gerstmann, C. Gougoussis, A. Kokalj, M. Lazzeri, L. Martin-Samos, N. Marzari, F. Mauri, R. Mazzarello, S. Paolini, A. Pasquarello, L. Paulatto, C. Sbraccia, S. Scandolo, G. Sclauzero, A.P. Seitsonen, A. Smogunov, P. Umari, R.M. Wentzcovitch, QUANTUM ESPRESSO: a modular and open-source software project for quantum simulations of materials, J. Phys. Condens. Matter 21 (2009) 395502-395519.

[30] R. Petrucci, P. Astolfi, L. Greci, O. Firuzi, L. Saso, G. Marrosu, A spectroelectrochemical and chemical study on oxidation of hydroxycinnamic acids in aprotic medium, Electrochim. Acta 52 (2007) 2461-2470.

[31] R. Petrucci, L. Saso, V. Kumar, A. Prasad, S.V. Malhotra, V. Parmar, G. Marrosu, A spectroelectrochemical and chemical study on oxidation of 7,8-dihydroxy-4-methylcoumarin (DHMC) and some related compounds in aprotic medium, Biochimie 92 (2010) 1123-1129.

[32] R. Greef, R. Peat, L.M. Peter, D. Pletcher, J. Robinson, Instrumental methods in Electrochemistry, Ch. 6, Ellis Horwood Limited, Chichester, England, 1985.

[33] R.S. Nicholson, I. Shain, Theory of stationary electrode polarography. Single scan and cyclic methods applied to reversible, irreversible, and kinetic systems, Anal. Chem. 36 (1964) 706-723.

[34] A. Trani, R. Petrucci, G. Marrosu, D. Zane, A. Curulli, Selective electrochemical determination of caffeine at a gold-chitosan nanocomposite sensor: may little change on nanocomposites synthesis affect selectivity?, J. Electroanal. Chem. 788 (2017) 99-106.

[35] B.H. Hansen, G. Dryhurst, Electrochemical oxidation of theophylline at the pyrolitic graphite electrode, J. Electroanal. Chem. Interfacial Electrochem. 32 (1971) 405-414.

[36] D.T. Sawyer, A. Sobkowiak, J.L. Roberts Jr, Electrochemistry for Chemists, John Wiley \& Sins, Inc., New York, 1995.

[37] L. Eberson, Electron-transfer reactions in organic chemistry, Adv. Phys. Org. Chem. 18 (1982) 79-185. 
A computational analyses based on the DFT was performed on CAF and TPh molecular structure: the ground state configurations of CAF and TPh were shown in Fig. 4, and the relevant bond distances and angles of both studied compounds were reported in Table S1.

Some differences regarding the penta-cyclic moiety were also evidenced by computational analysis of CAF and TPh molecular structure: a) the angles distortion, particularly affecting the angles with $\mathrm{N}^{7}$ and $\mathrm{C}^{8}$ at the vertices (Table S1), b) the density of states (DOS) of the last oc- cupied MO, with HOMO-1 and HOMO-2 of TPh shifted of $50 \mathrm{meV}$ upwards with respect to CAF (Fig. 5), and above all c) the PDOS HOMO decomposition (Fig. 6), evidencing that the lowest energy electrons of CAF were spread on the hexa-cyclic moiety with a large contribute from the $\mathrm{C}^{4}-\mathrm{C}^{5}$ bond, while the lowest energy electrons of $\mathrm{TPh}$ were shared with the penta-cyclic moiety with a large contribute from both $\mathrm{N}^{7}$ and $\mathrm{C}^{8}$ atoms (Section 3.3). This analysis supported the electrochemical data suggesting that different oxidative pathways might occur for CAF (and likely $\mathrm{TBr}$ ) and $\mathrm{TPh}$. 